\title{
THE MISSING LINK: MERGING NEUTRON STARS NATURALLY PRODUCE JET-LIKE STRUCTURES AND CAN POWER SHORT GAMMA-RAY BURSTS
}

\author{
Luciano Rezzolla ${ }^{1}$, Bruno Giacomazzo ${ }^{2,3}$, Luca Baiotti ${ }^{4}$, Jonathan Granot ${ }^{5}$, Chryssa Kouveliotou $^{6}$, \\ And Miguel A. Aloy ${ }^{7}$ \\ ${ }^{1}$ Max-Planck-Institut für Gravitationsphysik, Albert-Einstein-Institut, Potsdam, Germany \\ ${ }^{2}$ Department of Astronomy, University of Maryland, College Park, MD, USA \\ ${ }^{3}$ Gravitational Astrophysics Laboratory, NASA Goddard Space Flight Center, Greenbelt, MD, USA \\ ${ }^{4}$ Institute of Laser Engineering, Osaka University, Suita, Japan \\ ${ }^{5}$ Centre for Astrophysics Research, University of Hertfordshire, College Lane, Hatfield, Herts, AL10 9AB, UK \\ ${ }^{6}$ Space Science Office, VP62, NASA/Marshall Space Flight Center, Huntsville, AL 35812, USA \\ ${ }^{7}$ Departamento de Astronomía y Astrofisíca, Universidad de Valencia, 46100-Burjassot (Valencia), Spain \\ Received 2011 January 21; accepted 2011 March 2; published 2011 April 7
}

\begin{abstract}
Short gamma-ray bursts (SGRBs) are among the most luminous explosions in the universe, releasing in less than one second the energy emitted by our Galaxy over one year. Despite decades of observations, the nature of their "central engine" remains unknown. Considering a binary of magnetized neutron stars and solving the Einstein equations, we show that their merger results in a rapidly spinning black hole surrounded by a hot and highly magnetized torus. Lasting over $35 \mathrm{~ms}$ and much longer than previous simulations, our study reveals that magnetohydrodynamical instabilities amplify an initially turbulent magnetic field of $\sim 10^{12} \mathrm{G}$ to produce an ordered poloidal field of $\sim 10^{15} \mathrm{G}$ along the black hole spin axis, within a half-opening angle of $\sim 30^{\circ}$, which may naturally launch a relativistic jet. The broad consistency of our ab initio calculations with SGRB observations shows that the merger of magnetized neutron stars can provide the basic physical conditions for the central engine of SGRBs.
\end{abstract}

Key words: black hole physics - gamma-ray burst: general - gravitational waves - magnetohydrodynamics (MHD) - methods: numerical - stars: neutron

\section{INTRODUCTION}

The numerical investigation of the inspiral and merger of binary neutron stars (NSs) in full general relativity has made big strides in recent years. Crucial improvements in the formulation of the equations and numerical methods, along with increased computational resources, have extended the scope of early simulations. These developments have made it possible to compute the full evolution, from large binary separations up to black-hole (BH) formation, without and with magnetic fields (Shibata \& Taniguchi 2006; Baiotti et al. 2008; Anderson et al. 2008; Liu et al. 2008; Giacomazzo et al. 2009, 2011), and with idealized or realistic equations of state (EOS; Rezzolla et al. 2010; Kiuchi et al. 2010). This tremendous progress is also providing information about the entire gravitational waveform, from the early inspiral up to the ringing of the $\mathrm{BH}$ (see, e.g., Duez 2010; Baiotti et al. 2010). Advanced interferometric detectors starting from 2014 are expected to observe these sources at a rate of $\sim 40-400$ events per year (Abadie et al. 2010).

These simulations also probe whether the end product of mergers can serve as the "central engine" of short gamma-ray bursts (SGRBs; Paczynski 1986; Eichler et al. 1989; Narayan et al. 1992). The prevalent scenario invoked to explain SGRBs involves the coalescence of a binary system of compact objects, e.g., a BH and an NS or two NSs (Ruffert \& Janka 1999; Rosswog et al. 2003; Nakar 2007; Lee \& Ramirez-Ruiz 2007). After the coalescence, the merged object is expected to collapse to a BH surrounded by an accretion torus. An essential ingredient in this scenario is the formation of a central engine, which is required to launch a relativistic outflow with an energy of $\sim 10^{48}-10^{50}$ erg on a timescale of $\sim 0.1-1$ s (Nakar 2007; Lee \& Ramirez-Ruiz 2007). With only one possible exception (De Pasquale et al. 2010), SGRB afterglows do not clearly show a jet-associated light-curve steepening (Nakar 2007), thus suggesting typical jet opening half-angles of at least several degrees.

The qualitative scenario described above is generally supported by the association of SGRBs with old stellar populations, distinct from the young massive star associations for long GRBs (Fox et al. 2005; Prochaska et al. 2006). It is also supported to a good extent by fully general-relativistic simulations, which show that the formation of a torus of mass $M_{\text {tor }} \lesssim 0.4 M_{\odot}$ around a BH with spin $J / M^{2} \simeq 0.7-0.8$ is inevitable (Rezzolla et al. 2010). However, the simulations have so far failed to show that a jet can be produced. We here provide the first evidence that the merger of a binary of modestly magnetized NSs naturally forms many of the conditions needed to produce a jet of ultrastrong magnetic field, with properties that are broadly consistent with SGRB observations.

\section{NUMERICAL SIMULATIONS}

For the simulations we use the Cactus/Carpet/Whisky codes (Schnetter et al. 2004; Thornburg 2004; Giacomazzo \& Rezzolla 2007; Pollney et al. 2007) and we consider a configuration that could represent the properties of an NS binary a few orbits before its coalescence, within a fully general relativistic and an ideal-magnetohydrodynamic (MHD) framework (Giacomazzo \& Rezzolla 2007, 2011). More specifically, we simulate two equal-mass NSs, each with a gravitational mass of $1.5 M_{\odot}$ (i.e., sufficiently large to produce a $\mathrm{BH}$ soon after the merger), an equatorial radius of $13.6 \mathrm{~km}$, and on a circular orbit with initial separation of $\simeq 45 \mathrm{~km}$ between the centers (all length scales are coordinate scales; Taniguchi \& Gourgoulhon 2002). Confined in each star is a poloidal magnetic field with a maximum strength of $10^{12} \mathrm{G}$ 

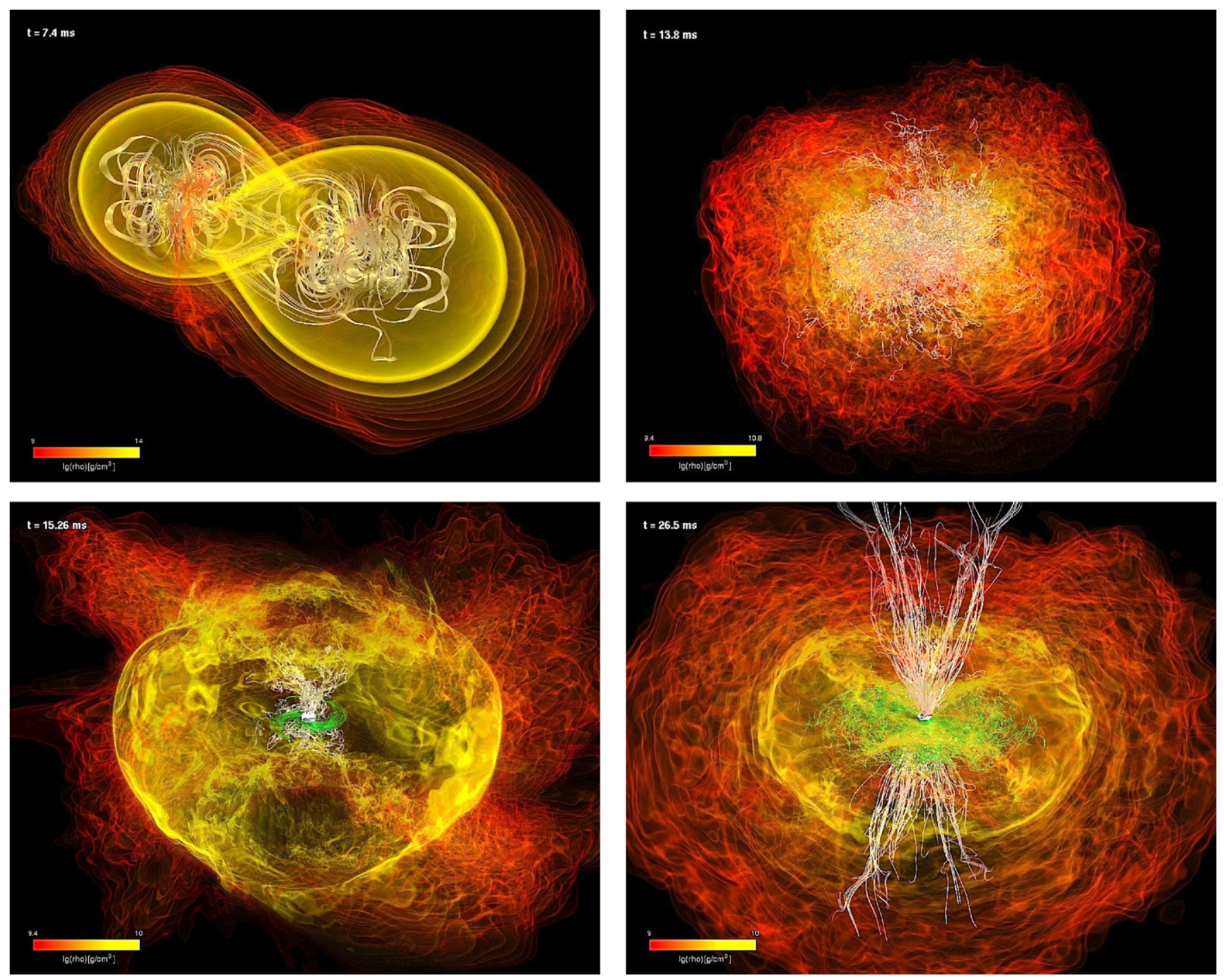

Figure 1. Snapshots at representative times of the evolution of the binary and of the formation of a large-scale ordered magnetic field. Shown with a color-code map is the density, over which the magnetic-field lines are superposed. The panels in the upper row refer to the binary during the merger $(t=7.4 \mathrm{~ms})$ and before the collapse to $\mathrm{BH}(t=13.8 \mathrm{~ms})$, while those in the lower row to the evolution after the formation of the $\mathrm{BH}(t=15.26 \mathrm{~ms}, t=26.5 \mathrm{~ms})$. Green lines sample the magnetic field in the torus and on the equatorial plane, while white lines show the magnetic field outside the torus and near the $\mathrm{BH}$ spin axis. The inner/outer part of the torus has a size of $\sim 90 / 170 \mathrm{~km}$, while the horizon has a diameter of $\simeq 9 \mathrm{~km}$.

(indicated as M1.62-B12 in Giacomazzo et al. 2011). At this separation, the binary loses energy and angular momentum via emission of gravitational waves (GWs), thus rapidly proceeding on tighter orbits as it evolves. After about $8 \mathrm{~ms}$ ( $\sim 3$ orbits), the two NSs merge forming a hypermassive NS (HMNS), namely, a rapidly and differentially rotating NS, whose mass, $3.0 M_{\odot}$, is above the maximum mass, $2.1 M_{\odot}$, allowed with uniform rotation by our ideal-gas $\mathrm{EOS}^{8}$ with an adiabatic index of 2 . Being metastable, an HMNS can exist as long as it is able to resist against collapse via a suitable redistribution of angular momentum (e.g., deforming into a "bar" shape; Shibata \& Taniguchi 2006; Baiotti et al. 2008), or through the pressure support coming from the large temperature increase produced by the merger. However, because the HMNS is also losing angular momentum through GWs, its lifetime is limited to a few ms, after which it collapses to a BH with mass $M=2.91 M_{\odot}$ and spin $J / M^{2}=0.81$, surrounded by a hot and dense torus with mass $M_{\text {tor }}=0.063 M_{\odot}($ Giacomazzo et al. 2011).

\footnotetext{
The use of a simplified EOS does not particularly influence our results besides determining the precise time when the HMNS collapses to a BH.
}

\section{DYNAMICS OF MATTER AND MAGNETIC FIELDS}

These stages of the evolution can be seen in Figure 1, which shows snapshots of the density color-coded between $10^{9}$ and $10^{10} \mathrm{~g} \mathrm{~cm}^{-3}$, and of the magnetic-field lines (green on the equatorial plane and white outside the torus). Soon after the BH formation the torus reaches a quasi-stationary regime, during which the density has maximum values of $\sim 10^{11} \mathrm{~g} \mathrm{~cm}^{-3}$, while the accretion rate settles to $\dot{M} \simeq 0.2 M_{\odot} \mathrm{s}^{-1}$. Using the measured values of the torus mass and of the accretion rate, and assuming the latter will not change significantly, such a regime could last for $t_{\text {accr }}=M_{\text {tor }} / \dot{M} \simeq 0.3 \mathrm{~s}$, after which the torus is fully accreted; furthermore, if the two NSs have unequal masses, tidal tails are produced which provide additional latetime accretion (Rezzolla et al. 2010). This accretion timescale is close to the typical observed SGRB durations (Kouveliotou et al. 1993; Nakar 2007). It is also long enough for the neutrinos produced in the torus to escape and annihilate in its neighborhood; estimates of the associated energy deposition rate range from $\sim 10^{48} \mathrm{erg} \mathrm{s}^{-1}$ (Dessart et al. 2009) to $\sim 10^{50} \mathrm{erg} \mathrm{s}^{-1}$ (Setiawan et al. 2004), thus leading to a total energy deposition 

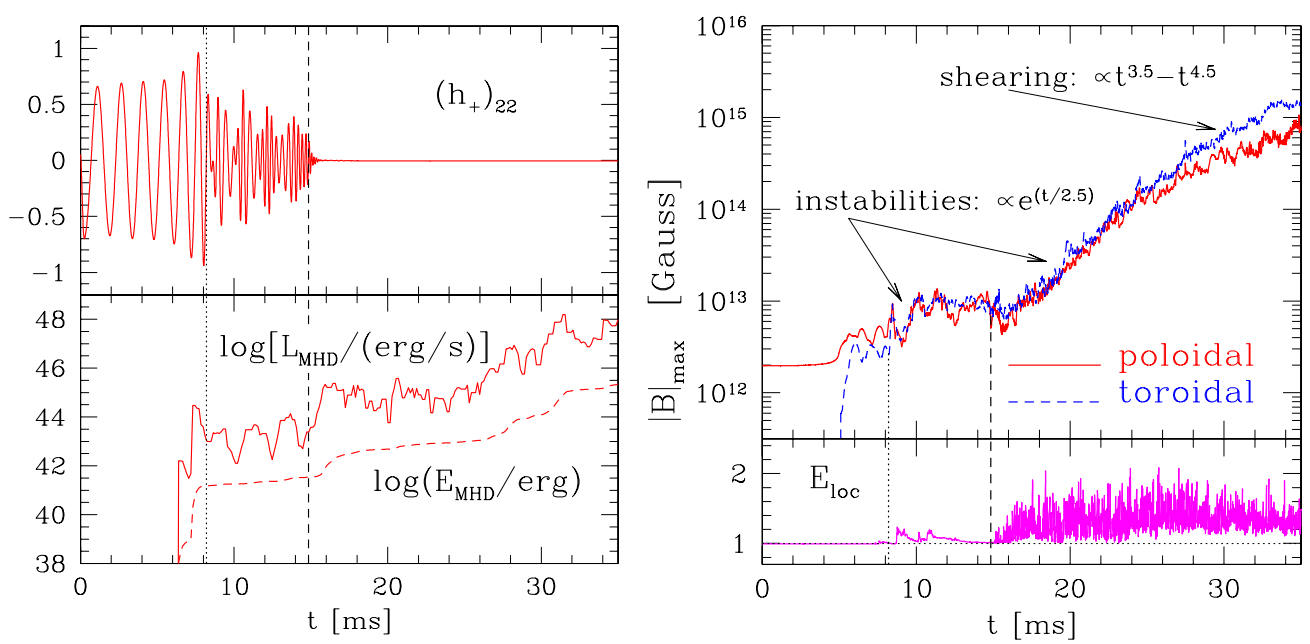

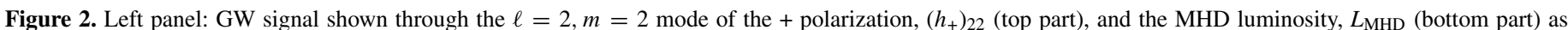

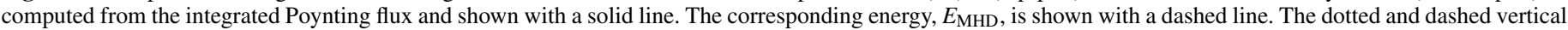

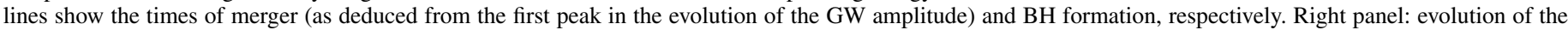

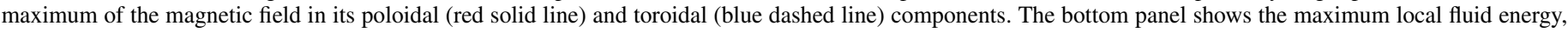
indicating that an unbound outflow (i.e., $E_{\text {loc }}>1$ ) develops and is sustained after $\mathrm{BH}$ formation.

between a few $10^{47}$ erg and a few $10^{49}$ erg over a fraction of a second. This energy would be sufficient to launch a relativistic fireball, but because we do not yet account for radiative losses, the large reservoir of thermal energy in the torus cannot be extracted in our simulations.

The GW signal of the whole process is shown in the left panel of Figure 2, while the bottom part exhibits the evolution of the MHD luminosity, $L_{\mathrm{MHD}}$, as computed from the integrated Poynting flux (solid line) and of the corresponding energy, $E_{\mathrm{MHD}}$ (dashed line). Clearly, the MHD emission starts only at the time of merger and increases exponentially after BH formation, when the GW signal essentially shuts off. Assuming that the quasi-stationary MHD luminosity is $\simeq 4 \times 10^{48} \mathrm{erg} \mathrm{s}^{-1}$, the total MHD energy released during the lifetime of the torus is $\simeq 1.2 \times 10^{48} \mathrm{erg}$, which, if spread over an opening half-angle of $\sim 30^{\circ}$ (see discussion below), suggests a lower limit to the isotropic equivalent energy in the outflow of $\simeq 9 \times 10^{48} \mathrm{erg}$. While this is at the low end of the observed distribution of gamma-ray energies for SGRBs, larger MHD luminosities are expected either through the additional growth of the magnetic field via the ongoing winding of the field lines in the disk (the simulation covers only one-tenth of $t_{\mathrm{accr}}$ ), or when magnetic reconnection (which cannot take place within our ideal-MHD approach) is also accounted for (which may also increase the gamma-ray efficiency; see, e.g., McKinney \& Uzdensky 2010).

The last two panels of Figure 1 offer views of the accreting torus after the $\mathrm{BH}$ formation. Although the matter dynamics is quasi-stationary, the last two panels clearly show that the magnetic field is not and instead evolves significantly. Only if the system is followed well after the formation of a $\mathrm{BH}$, MHD instabilities are seen to develop and generate the central, low-density, poloidal-field funnel. This regime, which was not accessible to previous simulations (Price \& Rosswog 2006; Anderson et al. 2008; Liu et al. 2008), is essential for the jet formation (Aloy et al. 2005; Komissarov et al. 2009). Because the strongly magnetized matter in the torus is highly conductive, it shears the magnetic-field lines via differential rotation. A measurement of the angular velocity in the torus indicates that it is essentially Keplerian and thus unstable to the magnetorotational instability (MRI; Balbus \& Hawley
1998), which develops $\simeq 5 \mathrm{~ms}$ after BH formation and amplifies exponentially both the poloidal and the toroidal magnetic fields; the $e$-folding time of the instability is $\simeq 2.5 \mathrm{~ms}$ and in good agreement with the one expected in the outer parts of the torus (Balbus \& Hawley 1998). Because of this exponential growth, the final value of the magnetic field is largely insensitive to the initial strength and thus a robust feature of the dynamics.

A quantitative view of the magnetic-field growth is shown in the right panel of Figure 2, which shows the evolution of the maximum values in the poloidal and toroidal components. Note that the latter is negligibly small before the merger, reaches equipartition with the poloidal field as a result of a Kelvin-Helmholtz instability triggered by the shearing of the stellar surfaces at merger (Price \& Rosswog 2006; Giacomazzo et al. 2009), and finally grows to $\simeq 10^{15} \mathrm{G}$ by the end of the simulation. At later times $(t \gtrsim 22 \mathrm{~ms}$ ), when the instability is suppressed, the further growth of the field is due to the shearing of the field lines and it increases only as a power law with exponent 3.5(4.5) for the poloidal (toroidal) component. Although the magnetic-field growth essentially stalls after $t \simeq 35 \mathrm{~ms}$, further slower growths are possible (Obergaulinger et al. 2009), yielding correspondingly larger Poynting fluxes. Indeed, when the ratio between the magnetic flux across the horizon and the mass accretion rate becomes sufficiently large, a Blandford-Znajek mechanism (Blandford \& Znajek 1977) may be ignited (Komissarov \& Barkov 2009); such conditions are not met over the timescale of our simulations, but could develop over longer timescales. Also shown in the right panel of Figure 2 is the maximum local fluid energy, highlighting that an unbound outflow (i.e., $E_{\mathrm{loc}}>1$ ) develops after $\mathrm{BH}$ formation along the outer walls of the torus and persists for the whole duration of the simulation.

Finally, Figure 3 provides a summary of the magnetic-field dynamics. It shows the magnetic field in the HMNS formed after the merger and its structure and dynamics after the collapse to $\mathrm{BH}$. In particular, in the last three panels it shows the magneticfield structure inside the torus and on the equatorial plane (green), and outside the torus and near the axis (white). It is apparent that the highly turbulent magnetic field in the HMNS $(t=13.8 \mathrm{~ms})$ changes systematically as the $\mathrm{BH}$ is produced 

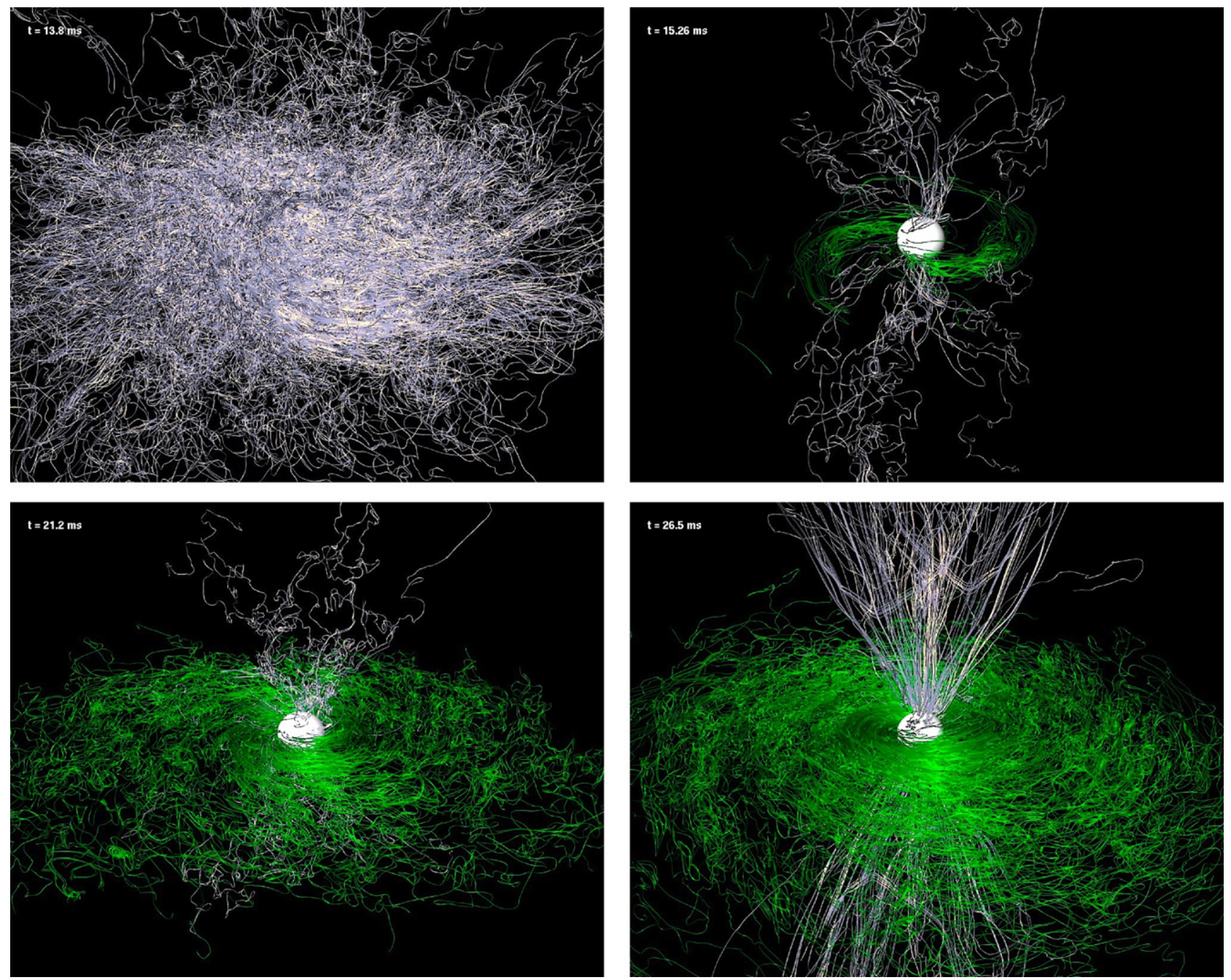

Figure 3. Magnetic-field structure in the HMNS (first panel) and after the collapse to BH (last three panels). Green refers to magnetic-field lines inside the torus and on the equatorial plane, while white refers to magnetic-field lines outside the torus and near the axis. The highly turbulent, predominantly poloidal magnetic-field structure in the HMNS $(t=13.8 \mathrm{~ms})$ changes systematically as the BH is produced ( $t=15.26 \mathrm{~ms})$, leading to the formation of a predominantly toroidal magnetic field in the torus $(t=21.2 \mathrm{~ms})$. All panels have the same linear scale, with the horizon diameter being of $\simeq 9 \mathrm{~km}$.

$(t=15.26 \mathrm{~ms})$, leading to the formation of a toroidal magnetic field in the torus $(t=21.2 \mathrm{~ms})$. As the MRI sets in, the magnetic field is not only amplified, but also organizes itself into a dual structure, which is mostly toroidal in the accretion torus with $B_{\text {tor }} \simeq 2 \times 10^{15} \mathrm{G}$, but predominantly poloidal and jet-like along the BH spin axis, with $B_{\mathrm{pol}} \simeq 8 \times 10^{14} \mathrm{G}(t=26.5 \mathrm{~ms})$. Note that the generation of an ordered large-scale field is far from trivial and a nonlinear dynamo may explain why the MRI brings a magnetic field self-organization, as it has been also suggested in case of MRI-mediated growth of the magnetic field in the conditions met in the collapse of massive stellar cores (Lesur \& Ogilvie 2008; Obergaulinger et al. 2009). However, the jetlike structure produced in the simulation is not yet the highly collimated ultrarelativistic outflow expected in SGRBs (see also below).

The hollow jet-like magnetic structure has an opening halfangle of $\sim 30^{\circ}$, which sets an upper limit for the opening halfangle of any potential outflow, either produced by neutrino energy deposition (Aloy et al. 2005) or by electromagnetic (EM) processes (Komissarov et al. 2009). In our simulations most of the outflow develops along the edges of the jet-like structure, via a turbulent layer of EM-driven matter, which shields the central funnel from excessive baryonic pollution. We envision that such a layer is crucial to set the opening angle of any ultrarelativistic jet, to shape both the radial and transverse structure of the jet, as well as to determine its stability properties. The Lorentz factors of the outflow measured in our simulations are not very high $(\Gamma \lesssim 4)$, but can potentially be amplified by several orders of magnitude in the inner baryon-poor regions through special-relativistic effects (Aloy \& Rezzolla 2006) or the variability of the flow (Granot et al. 2011). We expect that such accelerations will be produced as a more realistic and generalrelativistic treatment of the radiative losses will become computationally affordable.

\section{COMPARISON WITH OBSERVATIONS}

Below we briefly discuss how our results broadly match the properties of the central engine as deduced from the observations.

\subsection{Duration}

The observed duration of the prompt gamma-ray emission GRBs is energy dependent and is usually determined through $T_{x}$, the time over which $x \%$ of the total counts are observed, between the $(100-x) / 2$ and $(100+x) / 2$ percentiles. The most common 
intervals used are $T_{90}$ (or $T_{50}$ ), initially defined (Kouveliotou et al. 1993) between $20 \mathrm{keV}$ and $2 \mathrm{MeV}$. The GRB duration distribution is bimodal (Kouveliotou et al. 1993), where the durations of SGRBs (approximately $25 \%$ of GRBs) are well fit by a fairly wide log-normal distribution centered around $T_{90} \approx 0.8 \mathrm{~s}$ with an FWHM of $1.4 \mathrm{dex}$ (Nakar 2007). The typical redshifts of the SGRBs observed with Swift are in the range $z \sim 0.3-1$, suggesting a central value of the intrinsic duration distribution of $\approx(1+z)^{-1} 0.8 \mathrm{~s} \sim 0.5 \mathrm{~s}$ and a comparably wide distribution around this value. This is in close agreement with our accretion time of $\sim 0.3 \mathrm{~s}$.

\subsection{Energy}

The isotropic equivalent energy output in the prompt gammaray emission of SGRBs, $E_{\gamma}$,iso, spans a wide range, from $(2.7 \pm 1) \times 10^{48} \mathrm{erg}$ (in the observed energy range $\left.15-350 \mathrm{keV}\right)$ for GRB 050509B at a redshift of $z=0.225$ (Bloom et al. 2006) up to $(1.08 \pm 0.06) \times 10^{53} \mathrm{erg}$ (in the observed energy range $10 \mathrm{keV}-30 \mathrm{GeV}$ ) for GRB 090510 at $z=0.903$ (Ackermann et al. 2010). However, the most typical values are in the range $E_{\gamma, \text { iso }} \sim 10^{49}-10^{51}$ erg (Nakar 2007). In our model, the highly relativistic outflow may be powered either by neutrino-antineutrino annihilation, or by the Blandford-Znajek mechanism. For the former one might expect a total energy release between a few $10^{47}$ erg and $\sim 10^{49}$ erg (Oechslin et al. 2006; Birkl et al. 2007), into a bipolar relativistic jet of opening half-angle $\theta_{\text {jet }} \sim 8^{\circ}-30^{\circ}$, corresponding to a fraction $f_{b} \sim 0.01-0.13$ of the total solid angle, and isotropic equivalent energies, $E_{v \bar{v}}$,iso, between a few $10^{48} \mathrm{erg}$ and $\sim 10^{51} \mathrm{erg}$. For the latter mechanism, instead, and if the magnetization near the event horizon becomes sufficiently high, we could expect a jet power for our values for the BH mass and spin that is of (Lee et al. 2000; Pérez-Ramírez et al. 2010)

$$
L_{\mathrm{B}-\mathrm{Z}} \simeq 3.0 \times 10^{50}\left(\frac{f_{\mathrm{rel}}}{0.1}\right)\left(\frac{B}{2 \times 10^{15} \mathrm{G}}\right)^{2} \mathrm{erg} \mathrm{s}^{-1},
$$

where $f_{\text {rel }}$ is the fraction of the total Blandford-Znajek power that is channeled into the resulting relativistic jet (and $f_{\text {rel }} \sim 0.1$ might be expected for ejecta with asymptotic Lorentz factors above 100). This relativistic outflow is launched over a timescale of $\sim 0.2 \mathrm{~s}$ and corresponds to

$$
E_{\mathrm{B}-\mathrm{Z}, \text { iso }} \simeq 1.2 \times 10^{51}\left(\frac{f_{\mathrm{rel}}}{0.1}\right)\left(\frac{f_{b}}{0.05}\right)^{-1}\left(\frac{B}{2 \times 10^{15} \mathrm{G}}\right)^{2} \mathrm{erg} .
$$

Comparing the X-ray afterglow luminosity (after 10 or $11 \mathrm{hr}$ ) and $E_{\gamma \text {,iso }}$ suggests that the efficiency of the prompt gamma-ray emission in SGRBs is typically high (Bloom et al. 2006; Gehrels et al. 2009), and similar to that of long GRBs (Granot et al. $2006)$, with $E_{\gamma \text {,iso }} \sim(0.1-0.9) E_{\text {iso }}$, radiating between $\sim 10 \%$ and $\sim 90 \%$ of the initial energy of the ultrarelativistic outflow. Therefore, our model is able to accommodate the observed $E_{\gamma \text {,iso }}$ values.

\subsection{Lorentz Factor}

The Fermi Gamma-Ray Space Telescope has detected GeV emission from SGRBs (Abdo et al. 2009), suggesting typical lower limits of $\Gamma_{\min } \sim 10^{2}-10^{3}$. In particular, $\Gamma_{\min } \approx 1200$ was obtained for GRB 090510 (Ackermann et al. 2010). However, a more realistic model (Granot et al. 2008) results in $\Gamma_{\text {min }}$ values lower by a factor of $\sim 3$ (Ackermann et al. 2011). Therefore, the central engine should be capable of producing outflow Lorentz factors of at least a few hundred. The fact that our simulation produces a strongly magnetized mildly relativistic outflow at angles near $\sim 30^{\circ}$ from the $\mathrm{BH}$ spin axis would help shield the inner region near the spin axis from excessive baryon loading, and thus assist in achieving high asymptotic Lorentz factors at large distance from the source, after the outflow in this region is triggered by neutrinos and/or the Blandford-Znajek mechanism.

\subsection{Jet Angular Structure}

This is poorly constrained by observations (even more so than for long GRBs). The only compelling case for a jet break in the afterglow light curve is for GRB 090510 (De Pasquale et al. 2010 ), which occurred very early on (after $\sim 1400 \mathrm{~s}$ ), and would thus imply an extremely narrow jet $\left(\theta_{\text {jet }} \sim 0.2-0.4\right)$ and modest true energy output in gamma rays $\left(\sim 10^{48} \mathrm{erg}\right)$. If this is indeed a jet break, it might correspond to a line of sight near a very narrow and bright core of a jet, which also has significantly wider wings. Observers with lines of sight along these wings would then see a much dimmer and more typical SGRB (Rossi et al. 2002; Peng et al. 2005; Racusin et al. 2009); without such wings, however, the observations would suggest a very large intrinsic and beaming-corrected event rate per unit volume. In most cases there are only lower limits on a possible jet break time (Nakar 2007 ), resulting in typical limits of $f_{b} \gtrsim 10^{-2}$ or $\theta_{\text {jet }} \gtrsim 8^{\circ}$. This is consistent with our expectation of $\theta_{\text {jet }} \sim 8^{\circ}-30^{\circ}$ for the ultrarelativistic ejecta capable of producing an SGRB (which would also imply a reasonable SGRB intrinsic event rate per unit volume).

\section{CONCLUSIONS}

The calculations reported here clearly demonstrate that a binary merger of two NSs inevitably leads to the formation of a relativistic jet-like and ultrastrong magnetic field, which could serve as a central engine for SGRBs. Because the magneticfield growth is exponential, the picture emerging from our simulations is rather general and applies equally even to mildly magnetized NSs. Overall, this work removes a significant earlier uncertainty as to whether such binary mergers can indeed produce the central engines of SGRBs. While the EM energy release is already broadly compatible with the observations, our simulations lack a proper treatment of the energy losses via photons and neutrinos, which can provide a fundamental contribution to the energy input necessary to launch the fireball and cool the torus (Setiawan et al. 2004; Dessart et al. 2009). This additional energy input, whose self-consistent inclusion in general relativity remains extremely challenging, may help to launch an ultrarelativistic outflow very early after the $\mathrm{BH}$ forms and to complete the picture of the central engine of an SGRB.

It is a pleasure to thank Michael Koppitz, Ian Hinder, and Neil Gehrels for useful input. Partial support comes from the DFG grant SFB/Transregio 7, by "CompStar," a Research Networking Programme of the European Science Foundation, by the MEXT Grant-in-Aid for Young Scientists (22740163), by the NASA grant number NNX09AI75G, and by the European Research Council grant number 259276-CAMAP. J.G. gratefully acknowledges a Royal Society Wolfson Research Merit Award. 


\section{REFERENCES}

Abadie, J., et al. 2010, Class. Quantum Grav., 27, 173001

Abdo, A. A., et al. 2009, ApJ, 707, 580

Ackermann, M., et al. 2010, ApJ, 716, 1178

Ackermann, M., et al. 2011, ApJ, 729, 114

Aloy, M. A., Janka, H., \& Müller, E. 2005, A\&A, 436, 273

Aloy, M. A., \& Rezzolla, L. 2006, ApJ, 640, L115

Anderson, Matthew, Hirschmann, E. W., Lehner, L., Liebling, S. L., Motl, P. M., Neilsen, D., Palenzuela, C., \& Tohline, J. E. 2008, Phys. Rev. Lett., 100, 191101

Baiotti, L., Damour, T., Giacomazzo, B., Nagar, A., \& Rezzolla, L. 2010, Phys Rev. Lett., 105, 261101

Baiotti, L., Giacomazzo, B., \& Rezzolla, L. 2008, Phys. Rev. D, 78, 084033

Balbus, S. A., \& Hawley, J. F. 1998, Rev. Mod. Phys., 70, 1

Birkl, R., Aloy, M. A., Janka, H. T., \& Müller, E. 2007, A\&A, 463, 51

Blandford, R. D., \& Znajek, R. L. 1977, MNRAS, 179, 433

Bloom, J. S., et al. 2006, ApJ, 638, 354

De Pasquale, M., et al. 2010, ApJ, 709, L146

Dessart, L., Ott, C. D., Burrows, A., Rosswog, S., \& Livne, E. 2009, ApJ, 690, 1681

Duez, M. D. 2010, Class. Quantum Grav., 27, 114002

Eichler, D., Livio, M., Piran, T., \& Schramm, D. N. 1989, Nature, 340, 126

Fox, D. B., et al. 2005, Nature, 437, 845

Gehrels, N., Ramirez-Ruiz, E., \& Fox, D. B. 2009, ARA\&A, 47, 567

Giacomazzo, B., \& Rezzolla, L. 2007, Class. Quantum Grav., 24, S235

Giacomazzo, B., Rezzolla, L., \& Baiotti, L. 2009, MNRAS, 399, L164

Giacomazzo, B., Rezzolla, L., \& Baiotti, L. 2011, Phys. Rev. D, 83, 044014

Granot, J., Cohen-Tanugi, J., \& do Couto e Silva, E. 2008, ApJ, 677, 92

Granot, J., Komissarov, S., \& Spitkovsky, A. 2011, MNRAS, 411, 1323

Granot, J., Königl, A., \& Piran, T. 2006, MNRAS, 370, 1946

Kiuchi, K., Sekiguchi, Y., Shibata, M., \& Taniguchi, K. 2010, Phys. Rev. Lett., 104, 141101

Komissarov, S. S., \& Barkov, M. V. 2009, MNRAS, 397, 1153
Komissarov, S. S., Vlahakis, N., Königl, A., \& Barkov, M. V. 2009, MNRAS, 394, 1182

Kouveliotou, C., Meegan, C. A., Fishman, G. J., Bhat, N. P., Briggs, M. S., Koshut, T. M., Paciesas, W. S., \& Pendleton, G. N. 1993, ApJ, 413, L101

Lee, H. K., Wijers, R. A. M. J., \& Brown, G. E. 2000, Phys. Rep., 325, 83

Lee, W. H., \& Ramirez-Ruiz, E. 2007, New J. Phys., 9, 17

Lesur, G., \& Ogilvie, G. I. 2008, MNRAS, 391, 1437

Liu, Y. T., Shapiro, S. L., Etienne, Z. B., \& Taniguchi, K. 2008, Phys. Rev. D, 78, 024012

McKinney, J. C., \& Uzdensky, D. A. 2010, MNRAS, submitted (arXiv:1011.1904)

Nakar, E. 2007, Phys. Rep., 442, 166

Narayan, R., Paczynski, B., \& Piran, T. 1992, ApJ, 395, L83

Obergaulinger, M., Cerdá-Durán, P., Müller, E., \& Aloy, M. A. 2009, A\&A, 498, 241

Oechslin, R., \& Janka, H. T. 2006, MNRAS, 368, 148

Paczynski, B. 1986, ApJ, 308, L43

Peng, F., Königl, A., \& Granot, J. 2005, ApJ, 626, 966

Pérez-Ramírez, D., et al. 2010, A\&A, 510, 105

Pollney, D., et al. 2007, Phys. Rev. D, 76, 124002

Price, R. H., \& Rosswog, S. 2006, Science, 312, 719

Prochaska, J. X., et al. 2006, ApJ, 642, 989

Racusin, J. L., et al. 2008, Nature, 455, 183

Rezzolla, L., Baiotti, L., Giacomazzo, B., Link, D., \& Font, J.-A. 2010, Class. Quantum Grav., 27, 114105

Rossi, E., Lazzati, D., \& Rees, M. J. 2002, MNRAS, 332, 945

Rosswog, S., Ramirez-Ruiz, E., \& Davies, M. B. 2003, MNRAS, 345, 1077

Ruffert, M., \& Janka, H.-Th. 1999, A\&A, 344, 573

Schnetter, E., Hawley, S. H., \& Hawke, I. 2004, Class. Quant. Grav., 21, 1465

Setiawan, S., Ruffert, M., \& Janka, H. 2004, MNRAS, 352, 753

Shibata, M., \& Taniguchi, K. 2006, Phys. Rev. D, 73, 064027

Taniguchi, K., \& Gourgoulhon, E. 2002, Phys. Rev. D, 66, 104019

Thornburg, J. 2004, Class. Quant. Grav., 21, 743 